\title{
Réflexions sur la construction de l'arbre généalogique avec des enfants ou des adolescents
}

\author{
CLAIRE METZ
}

Une technique d'entretien se répand actuellement, consistant à faire construire au sujet son arbre généalogique à l'aide de ses seuls souvenirs. Cet outil est également utilisé en psychologie clinique pour des entretiens de recherche, la visée est alors de recueillir le maximum d'informations. Ce dispositif s'est d'autant plus répandu que nous traversons une période de transformations familiales sans précédent, et que les nouvelles formes d'organisation familiale posent parfois, il est vrai, des problèmes de repérage. Les professionnels, eux-mêmes, sont confrontés à ces difficultés de repérage des places généalogiques et des liens d'alliance et de parenté. L'idée est véhiculée selon laquelle cet instrument ne présente que des avantages car il permet au sujet de mieux se situer dans son identité personnelle et familiale. Cependant, il nous semble que ces pratiques méritent réflexion, selon le cadre où elles sont utilisées et selon les personnes à qui elles s'adressent.
Quels sont les enjeux de cette technique d'intervention ? Que mobilise-t-elle, en particulier, chez l'enfant ou l'adolescent qui construit son arbre généalogique ? Est-elle un outil technique fécond pour les professionnels et structurant pour les enfants et adolescents qui s'y confrontent ? Nous distinguerons dans cette réflexion le génogramme utilisé dans une pratique clinique à visée thérapeutique, de son emploi dans un autre cadre. Nous nous référons à la psychanalyse pour élaborer ce questionnement.

\section{Donner la parole}

Telle que nous l'utilisons, nous définissons cette technique dite " génogramme » comme " une représentation graphique de la famille " (Lemaire-Arnaud, 1980, p. 33), faite de mémoire, sur plusieurs générations. Cependant, ce n'est pas tant sous la forme d'un résultat, d'un produit d'anamnèse, qu'il nous semble intéressant, mais en tant que procès 
incluant les affects qu'il provoque et le discours qui l'accompagne. Ainsi, nous cherchons à recueillir des données significatives pour le sujet plutôt que d'effectuer une démarche anamnestique exhaustive. Ce qui est important, c'est la façon dont l'auteur de cet arbre «fantasmatique » perçoit ses ascendants et les liens qui les unissent et qui le lient à ses ascendants et collatéraux et à leurs rôles (Ancelin-Schützenberger, 1993, p. 89). Cette construction donne la parole au sujet et laisse place à la dimension fantasmatique. Le professionnel, selon sa formation, peut travailler sur les fantasmes du sujet et/ou travailler sur l'ordonnancement des places généalogiques. Ce dernier point induit l'idée que la construction d'arbres généalogiques favorise la mise en place pour le sujet de repères familiaux, de temps et d'espace.

\section{Des données pour la recherche}

Que cette technique donne la parole au sujet, lui permette d'exprimer ses fantasmes et favorise les repères structurants, semble en faire un excellent outil. Cette mise à plat graphique permet de visualiser les données, ce qui a deux effets. Le sujet a sous les yeux ce qu'il a dit, ce qu'il n'a pas dit, ce qui peut l'inciter à compléter son arbre et l'entraîner à davantage d'exhaustivité. D'autre part, cette visualisation peut avoir un effet de révélation : des coïncidences, des inconnues, des répétitions, des traces de secrets sautent aux yeux. "L'arbre dessiné ici se différencie de l'œuvre du généalogiste en ce qu'il nous parle. À côté de rameaux feuillus se dessinent, dans l'ombre, quelques branches dénudées, indices de secrets et de mythes familiaux (...), la représentation graphique facilite la visualisation des patterns de répétition transgénérationnelle » (Cannels, 1993, p. 54). Cet effet se produit pour le sujet comme pour son interlocuteur, ce qui enrichit la conduite de l'entretien et les questions qui se posent. Cet outil « qui procure vite des données fondamentales au clinicien $»$ peut produire un effet de fascination sur les professionnels, " d'où la tentation de l'utiliser à temps et à contretemps » (LemaireArnaud, 1980, p. 38), de manière systématique, sans plus de réflexion sur la pertinence de sa pratique.

Cependant, compte tenu des avantages exposés, nous avions pensé à son utilisation dans la recherche, lors d'une étude portant sur les enfants et adolescents atteints de déficience intellectuelle légère. La construction de l'arbre généalogique présentait l'intérêt d'être un outil de médiation. Or, les entretiens avec les enfants nécessitent en général un médiateur.

Cette recherche d'orientation psychanalytique présentait une difficulté spécifique qu'évoque Maud Mannoni dans L'enfant arriéré et sa mère. En effet l'enfant dit " débile » parle difficilement car « il est parlé » (Mannoni, 1964, p. 175). La construction de l'arbre généalogique paraissait un dispositif fécond pour recueillir des données discursives, précisément autour de cette construction.

Ce support a effectivement permis aux enfants et adolescents ren- 
contrés pour cette recherche de s'impliquer. Les résultats ont été très intéressants et ont permis la formulation de nouvelles hypothèses. J'ai observé que le discours de l'enfant " débile " peut se figer brusquement, l'enfant s'arrête, annonce qu'il « ne sait pas », qu'il " a oublié ». La recherche met en évidence que ces arrêts du discours, loin de révéler la bêtise du sujet, indiquent les points de réticence, de souffrance ou les tabous liés à des éléments filiatifs de son histoire, que cette apparente bêtise lui permet de masquer.
J'ai développé le cas de Milena dans un article précédent (Metz, 1999). Je résume brièvement ce qui a trait au génogramme : sa construction mettait en évidence l'existence $\mathrm{du}$ secret familial portant sur le grand-père maternel camerounais noir. Le discours familial faisait croire à Milena que toute sa famille était blanche. Or, elle avait le teint foncé, les cheveux noirs et crépus. Pour croire au mensonge familial, il lui aurait fallu ne pas se voir. Voici le génogramme qu'avait construit Milena :

Génogramme de Milena

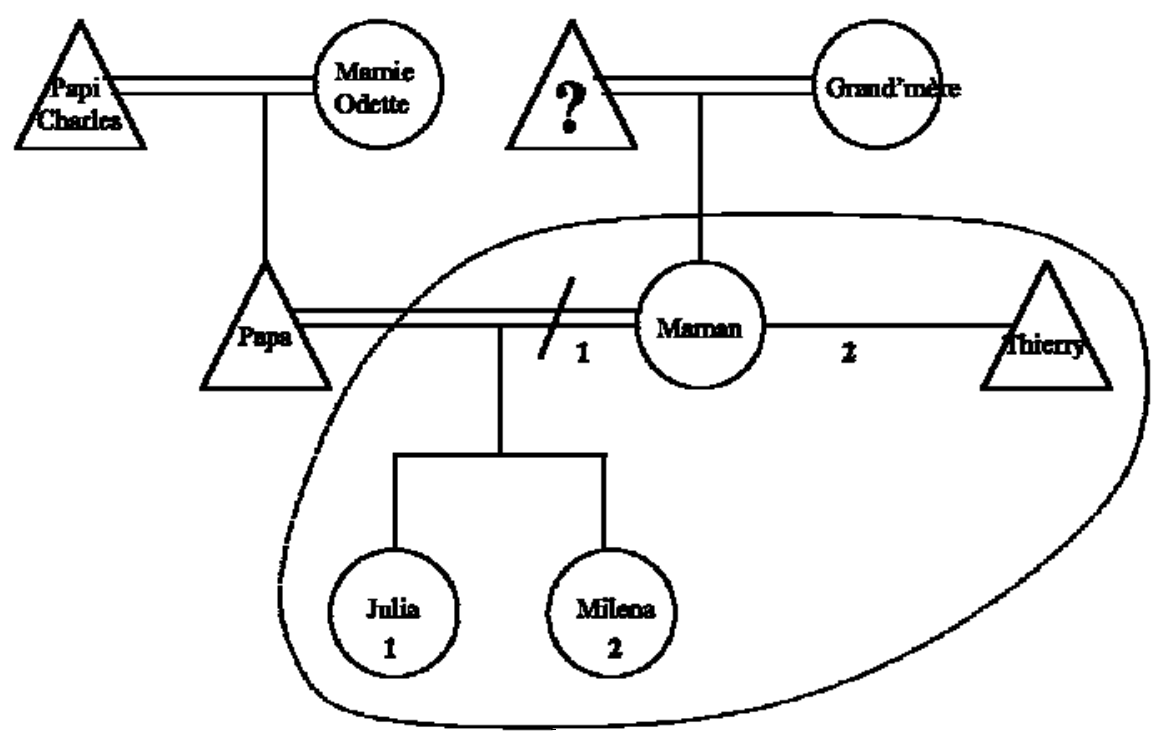

La comparaison avec le génogramme réel montrait son erreur : les liens de filiation étaient faux : papi Charles et mamie Odette étaient en réalité le beau-père et la mère de sa mère « Marie ».

J'avais montré que l'effet de cette erreur était de laisser vide la place du grand-père maternel dont rien n'était dit dans l'entretien. Or, c'était justement cette place qui faisait trou dans le savoir de Milena sur sa filiation. La case vide dans la construction du génogramme venait coïncider avec la case vide de la réalité dont Milena ignorait tout, et de 
Génogramme réel

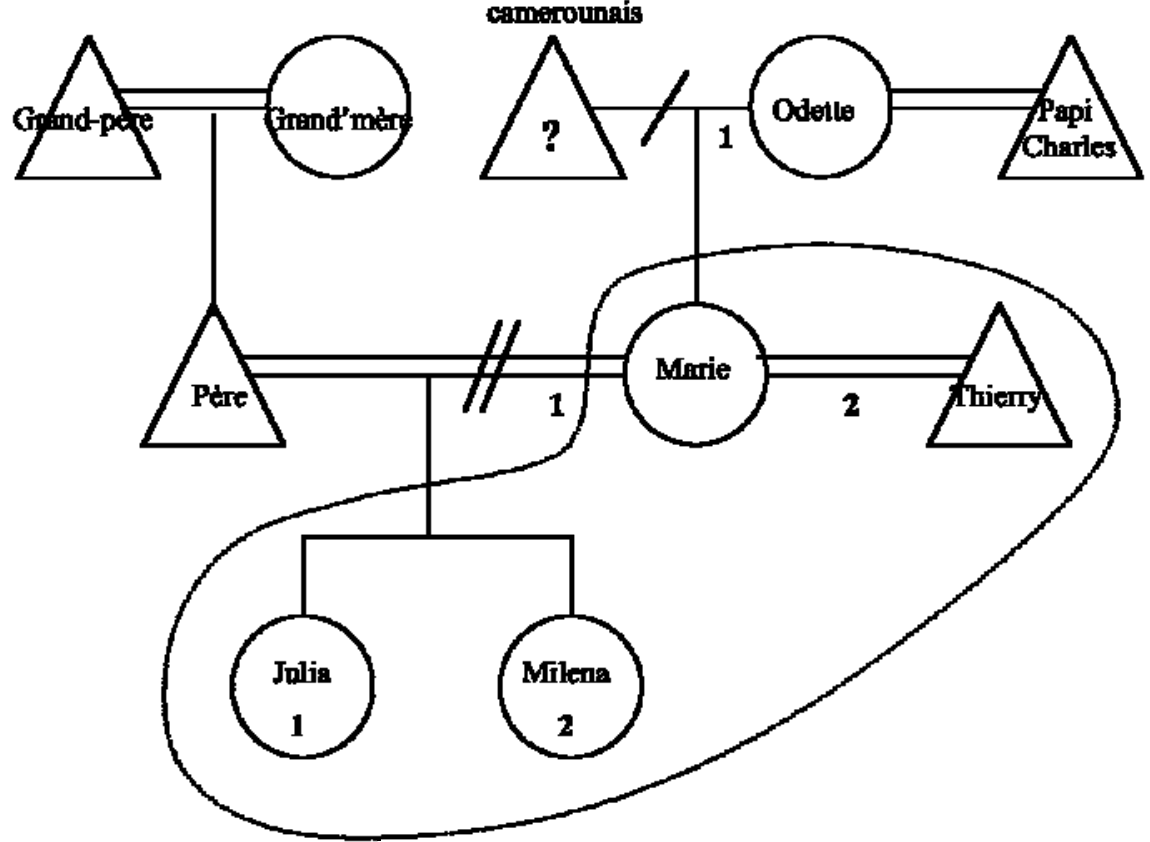

la personne et de l'existence même de cette case vide.

Je concluais alors aux bénéfices de cette méthode, pour le sujet comme pour le chercheur.

\section{Les points de souffrance}

Or, c'est sur la question des points de souffrance liés aux éléments filiatifs que cette pratique nécessite d'être interrogée, me semble-t-il. Je résume brièvement un cas provenant de cette même recherche, qui alertait déjà mon questionnement sur la pratique du génogramme (Metz, 2005).

Karima était une fillette qui avait été l'objet de confusions généalogiques importantes. Sa mère et ellemême vivaient auparavant au Maroc chez les grands-parents maternels de Karima et ses sept oncles et tantes, tandis que le père travaillait en France et ne rentrait que tous les deux ans. D'autre part, les liens de parenté n'avaient pas été clairement explicités à la fillette, si bien qu'elle croyait, jusqu'à son arrivée en France à l'âge de 4 ans, que ses grands-parents étaient ses parents et que sa mère était sa sœur.

Lors de la construction du génogramme, le père de Karima était passé sous silence, son discours restant très fuyant chaque fois que je lui en reparlais, de manière si répétitive que je lui demandais si elle n'aimait pas parler de son père. Elle répondit que ça lui faisait penser à son grandpère. Si Karima ne faisait plus de confusions généalogiques, je remar- 
quais que « papa » faisait immédiatement resurgir " grand-père ", c'est-à-dire celui qu'elle dénommait « papa » dans son enfance.

Lors de la suite de l'entretien, elle évoqua le Maroc. " $\mathrm{Au} \mathrm{Maroc,} \mathrm{le}$ sable est tout doux, ça me va bien au Maroc, ça ne me va pas bien ici. » Elle dit aussi qu' " au Maroc il y a une école pour apprendre ». Elle ajouta : « Je voulais rester. Le Dieu, il est aussi là-bas au Maroc. Mais il est dans le ciel. C'est bien au Maroc, ça fait pleurer », elle se mit à pleurer et, dans une grande émotion, elle évoqua son grand-père, concluant par ces mots : « J'espère que j'y vais. » Le génogramme avait confronté la fillette à une nostalgie profonde du Maroc, mais aussi à une perte irréparable, qui était cette construction familiale erronée, reposant sur des places généalogiques falsifiées, son grand-père en place de père, sa grand-mère en place de mère, et ses oncles et tantes en place de frères et sœurs. Il était clair que l'émotion avait submergé Karima, que j'avais consolée, et que cette technique ne me semblait plus si banale et si automatiquement avantageuse.

Après cette recherche, il se trouve que je m'engageai dans une pratique de psychothérapie analytique avec des enfants et des adolescents. Deux aspects problématiques me sont alors nettement apparus : la construction d'arbres généalogiques avec l'enfant et l'adolescent comporte un forçage car cet outil, s'il est utilisé sans précaution, est forcément intrusif, dans la mesure où il interroge la structure familiale. Ainsi, « ce travail est souvent l'occasion d'émergence d'affects qui peuvent être bouleversants " (Lemaire-Arnaud, 1980, p. 35). Un forçage est douloureux, mais de plus il ne respecte ni les chaînes associatives ni le temps nécessaire à l'élaboration psychique. En effet, la mise en place de repères familiaux est le produit d'une élaboration de l'histoire familiale et en ce sens requiert un travail de mentalisation.

À rompre ou négliger les chaînes associatives, les récits arrivent trop vite, l'enfant n'est pas prêt, les affects ne sont pas assez appropriés, et le sens qu'ils auraient pu prendre en les laissant venir est perdu pour un temps. Dans ce cas, l'effet de révélation que produit la visualisation des données peut n'avoir pas plus de sens que de révéler à un patient un désir inconscient. Ainsi, deux risques se dégagent : le bouleversement ou, au contraire, la perte de sens.

Il n'est donc pas certain que pratiquer la construction d'arbre généalogique favorise un repérage structurant pour le sujet. Se repérer dans ses liens familiaux, s'approprier son histoire familiale, tout cela nécessite un travail d'élaboration effectué au cours d'échanges langagiers dans le cadre d'un transfert, un travail d'accompagnement afin de pouvoir surmonter la souffrance liée à certains événements familiaux, aux liens ou aux absences de liens.

C'est pourquoi cette technique hors d'un cadre adéquat, par exemple trop rapidement utilisée dans un premier entretien, peut susciter cette souffrance au lieu de permettre de la surmonter. N'oublions pas que le tissu familial est actuellement, pour nombre d'enfants et 
d'adolescents, marqué de déchirures que les séparations ont opérées. Des années peuvent être nécessaires pour en effectuer le deuil, et la confrontation immédiate avec la représentation visuelle de cette famille au cours d'un entretien peut s'avérer bien douloureuse. Évelyne Lemaire-Arnaud (1980, p. 37), évoquant l'utilisation du génogramme dans un groupe de psychothérapeutes, le spécifiait très clairement : «Étant donné la lourdeur des affects soulevés et les retombées personnelles qu'une telle réflexion sur son propre tissu familial peut avoir sur chacun, des mesures de très grande prudence sont nécessaires avant de l'entreprendre. »

Utiliser cette technique requiert, en conséquence, l'établissement d'un cadre transférentiel et la prise en compte du travail d'élaboration psychique, particulièrement avec les enfants et adolescents. Ce travail de construction du génogramme est un médiateur certes, mais pour les raisons que j'ai développées, cette technique ne me semble pas pouvoir être utilisée comme d'autres médiations, telles que le jeu ou le dessin ni même les tests projectifs. En effet, aucun de ces moyens ne sollicite les faits de la réalité familiale aussi directement que la construction de l'arbre généalogique. Le génogramme est donc loin d'être une technique, simplement banale ou commode, qui permettrait d'engager un entretien dans une pratique courante de rencontres avec les familles.

Les travaux sur les faits trans- et intergénérationnels, en particulier ceux portant sur les secrets de famille, ont souligné l'importance des dynamiques relationnelles verticales. C'est pourquoi, cet outil conserve les intérêts que nous avons précisés, sous réserve d'être employé en temps utile, dans un cadre thérapeutique, lorsqu'une relation transférentielle est installée. Plusieurs cas peuvent se produire. Il arrive tout simplement qu'au cours d'une psychothérapie, le repérage graphique soit devenu nécessaire, du fait de la complexité des structures familiales. Le praticien peut d'ailleurs toujours 1'utiliser pour schématiser à part soi les données recueillies au fur et à mesure que le matériel verbal est donné par le sujet (Lemaire-Arnaud, 1980, p. 38).

Cependant, le génogramme, en tant que construction faite par le sujet, peut faire partie intégrante de la technique du thérapeute, qui l'emploie alors de manière à apporter l'accompagnement nécessaire pendant et après la construction, afin de permettre au sujet de traverser le moment de révélation que constitue cette représentation graphique. Et aussi de surmonter les effets ultérieurs de certaines prises de conscience qui peuvent se révéler bouleversantes. C'est ainsi que cet outil prend tout son sens dans sa dimension clinique. Évelyne Lemaire-Arnaud en donne un bon exemple dans le cas de Suzy, 3 ans, consultant pour spasmes du sanglot. La construction du génogramme met en évidence l'ensemble des aïeuls en adoration devant le bébé et usurpant le rôle des parents, et du côté paternel, l'absence de lignée faisant référence : « Tous des voleurs », selon le discours maternel. Les parents prennent conscience de cet ensemble 
avec beaucoup d'émotion et la relation transférentielle leur permet au cours des entretiens successifs de dénouer la situation (LemaireArnaud, 1980, p. 39-40).

Nous pouvons évoquer un parallèle avec l'image inconsciente du corps : cette image est le produit d'identifications, ce n'est pas le résultat d'un savoir sur les parties du corps ou d'un travail sur le schéma corporel. De même, la représentation de l'ordre généalogique familial n'est pas le résultat d'un savoir sur la représentation graphique de l'arbre généalogique, mais d'un travail d'élaboration. Nous pourrions en ce sens formuler l'idée que le génogramme, en tant qu'il sollicite une image inconsciente de la famille, nécessite un cadre thérapeutique.

Claire Metz Psychanalyste, psychologue clinicienne docteur en psychologie, membre du laboratoire de psychologie clinique : famille et filiation EA 3071

Strasbourg claire.metz@voila.fr

\section{BIBLIOGRAPHIE}

AnCElin-Schutzenberger, A. 1993. Aïe, mes aïeux !, Paris, La Méridienne, Desclée de Brouwer.

Assoun, P.-L. 1988. « Trouble de penser et pensée du trouble », Le trouble de penser, Nouvelle revue de psychanalyse, p. 77-106.

Cannels, M. 1993. «Un scénario, des acteurs et une mise en scène. Le génogramme et son processus », Dialogue, $\mathrm{n}^{\circ} 126$, p. 53-63.

Cordie, A. 1993. Les cancres n'existent pas, Paris, Le Seuil.
FreUd, S. 1987. Trois essais sur la théorie de la sexualité, Paris, Gallimard.

Lemaire-Arnaud, E. 1980. « À propos d'une technique nouvelle : le génogramme », Dialogue, n 70, p. 33-46.

Lemaire-Arnaud, E. 1988. « Le génogramme et les secrets de famille », Dialogue, $\mathrm{n}^{\circ} 100$, p. 106-110.

MaC Goldrick, M. ; Randy, G. 1990. Génogrammes et entretien familial, Paris, ESF.

Mannoni, M. 1964. L'enfant arriéré et sa mère, Paris, Le Seuil.

Metz, C. 1999. « Génogramme et handicap mental ou quand la "bêtise" d'un enfant masque des silences familiaux $»$, Dialogue, $n^{\circ} 145$, p. 51-62.

Metz, C. 2005. « Apprendre en exil : barrière culturelle et inhibition intellectuelle. L'enfant en exil ou l'enfant hors-lieu ",, Revue Enfances-adolescences.

Viaux, J.-L. 2002. L'enfant et le couple en crise. Du conflit psychologique au contentieux juridique, Paris, Dunod.

\section{RÉSUMÉ}

La construction de l'arbre généalogique tend à se répandre comme médiateur au cours d'entretiens dans des cadres aussi différents que le travail social, les enquêtes de recherche et le travail clinique thérapeutique. Mise en place de repères structurants pour l'identité singulière et familiale de la personne, outil de médiation facilitant la parole, cette technique semble présenter nombre d'avantages. Pourtant son utilisation avec des enfants ou des adolescents mérite d'être interrogée. En effet, la construction de l'arbre généalogique est un médiateur particulier car il sollicite directement les données familiales. Or, le repérage dans ces données est le fruit d'un travail d'élaboration qui nécessite un temps psychique et un cadre transférentiel qui ne peuvent être court-circuités.

MOTS-CLÉS

Arbre généalogique, médiateur, repères, temps psychique, transfert. 